\title{
Emotionale Framing-Effekte auf Einstellungen: Ein integratives Modell
}

\author{
Rinaldo Kühne
}

\begin{abstract}
Die Forschung zu Framing-Effekten auf Einstellungen fokussierte bisher kognitive Wirkungsprozesse. Medien-Frames regen aber nicht nur kognitive Urteilsprozesse an, sondern vermögen auch Emotionen auszulösen, die Implikationen für die Meinungsbildung haben. Im Beitrag wird auf Basis von Emotions- und Framing-Theorien ein integratives Modell kognitiver und emotionaler Wirkungsprozesse entwickelt. Das Modell basiert auf der Grundidee, dass aus der kognitiven Verarbeitung von Medien-Frames Emotionen resultieren können, welche die Einstellungen der Rezipienten beeinflussen.
\end{abstract}

Schlagwörter: Framing, Wirkungen auf Einstellungen, kognitive Prozesse, emotionale Prozesse

\section{Einleitung}

Die Rezeptions- und Wirkungsforschung fokussierte im Zuge der kognitiven Wende in der Psychologie traditionell kognitive Effekte der Mediennutzung. Zentrale kommunikationswissenschaftliche Wirkungstheorien wie der Agenda-Setting-, der Kultivierungs- und der Priming-Ansatz postulieren, dass kognitive Prozesse der Wirkung von Medieninhalten auf den Rezipienten zugrunde liegen (Schenk, 2007). Auch in der Framing-Forschung werden Wirkungen von Medien-Frames durch kognitive Prozesse erklärt. Postuliert wird etwa, dass Frames bestimmte Gedächtnisinhalte aktivieren sowie die Anwendbarkeit und Wichtigkeit kognitiver Konzepte erhöhen (vgl. Price \& Tewksbury, 1997; Nelson, Oxley \& Clawson, 1997).

Obschon bereits Zillmann ab den 1960er Jahren emotionstheoretische Überlegungen in die kommunikationswissenschaftliche Theoriebildung einfließen ließ (vgl. Bryant, Roskos-Ewoldsen \& Cantor, 2003), fanden diese vor allem in den letzten zwei Dekaden in größerem Umfang Eingang in Theorien der Rezeptions- und Wirkungsforschung (vgl. Wirth \& Schramm, 2005). Umso erstaunlicher ist, dass emotionale Wirkungen von Medien-Frames bislang kaum untersucht wurden. Erste Studien deuten darauf hin, dass das Framing von Nachrichtenbeiträgen Emotionen wie Mitleid oder Ärger auslösen kann, welche die Einstellungsbildung beeinflussen (z. B. Gross, 2008). Neben den empirischen Befunden sprechen weitere Gründe für die Untersuchung emotionaler Prozesse. Zum einen ist dies aus analytischen Gesichtspunkten relevant. Die genaue Aufschlüsselung von Wirkungsprozessen in kognitive und emotionale Teilprozesse sowie die Analyse ihres Zusammenspiels erhöhen den Erklärungsgehalt von Framing-Modellen. Dies gilt insbesondere, da kognitive und emotionale Prozesse unterschiedliche Resultate hervorbringen können und durch unterschiedliche Randbedingungen bzw. Moderatoren beeinflusst werden (vgl. Abschnitt 7). Zum anderen ist die Untersuchung emotionaler Prozesse aus normativen Erwägungen von Bedeutung: Emotionseinflüsse auf Meinungsbildungsprozesse werden oft kritisch beurteilt (Marcus, 2002). Kritisiert wird unter anderem, dass Emotionen die Entscheidungsautonomie von Individuen reduzieren, da sie sich der Kontrolle durch die Vernunft entziehen, und dass sie impulsive und unvernünftige Urteile begünstigen, die nicht auf der Abwägung von Argumenten basieren. $\mathrm{Da}$ aus normativen Gesichtspunkten also eher der Prozess der Meinungsbildung als das 
Resultat von Bedeutung ist, setzt eine normative Bewertung von Rezeptionsprozessen die Unterscheidung kognitiver und emotionaler Wirkungsprozesse voraus.

Der vorliegende Beitrag widmet sich der Frage, wie und unter welchen Bedingungen Medien-Frames Emotionen auslösen und welchen Einfluss diese Emotionen auf die Einstellungen der Rezipienten zu den berichteten Themen haben. Ausgehend von Emotionstheorien und Ansätzen zu kognitiven Wirkungen von Medien-Frames wird ein integratives Modell entwickelt. Die Kernidee des Modells ist, dass die kognitive Verarbeitung von Medien-Frames in bestimmten Fällen Emotionen auslösen kann, die in die Urteilsbildung einfließen. Der Beitrag ist folgendermaßen gegliedert: Zunächst wird ein Überblick zu kognitiven Wirkungen von Medien-Frames gegeben. Danach werden empirische Befunde zu emotionalen Wirkungen von Medien-Frames referiert. Im Hauptteil wird erörtert, wie bisherige Framing-Ansätze um emotionstheoretische Überlegungen ergänzt werden können, um emotionale Wirkungen von Frames zu erklären. Schließlich werden ein integriertes Modell und Forschungsdesiderate diskutiert.

\section{Begriffsbestimmungen}

Die Konzeptualisierung kognitiver und emotionaler Framing-Prozesse setzt eine Bestimmung der Begriffe Medien-Frame, Kognition und Emotion voraus. Ein MedienFrame kann laut Gamson und Modigliani als „central organizing idea or story line that provides meaning to an unfolding strip of events, weaving a connection among them" (1987: 143) oder als "set of interpretative packages that give meaning to an issue” (1989: 3) umschrieben werden. Medien-Frames können demnach als textliche Interpretationsrahmen oder Argumentationsmuster verstanden werden, die eine bestimmte Lesart eines Themas nahelegen (vgl. Matthes, 2007). Grundannahme ist dabei, dass gesellschaftspolitische Themen aufgrund ihrer Komplexität und Ambiguität aus unterschiedlichen Perspektiven betrachtet werden können. Verschiedene Frame-Konzeptionen verbindet die Idee, dass Medien-Frames nicht nur bestimmte Aspekte eines Themas hervorheben, sondern diese auch auf eine bestimmte Weise miteinander verknüpfen bzw. das Thema strukturieren (B. Scheufele, 2003; D. Scheufele \& Tewksbury, 2007; Pan \& Kosicki, 1993). So versteht B. Scheufele (2003) Frames als Interpretationsmuster, die aus mehreren Objekten und Objektrelationen bestehen. Die Deutung eines Themas beeinflussen Medien-Frames demzufolge über die Hervorhebung und insbesondere die Verknüpfung verschiedener Aspekte eines Themas. Verknüpfungen müssen dabei nicht explizit gemacht werden, sondern können auch nur implizit vorliegen (Pan \& Kosicki, 1993). Welche Aspekte eines Themas hervorgehoben und verknüpft werden können, erörtert Entman (1993). Laut Entman umfassen Frames bestimmte Problemdefinitionen, explizite Bewertungen, Ursachenzuschreibungen und Lösungszuschreibungen. Das heißt, Medien-Frames legen fest, was für ein Problem vorliegt, wie dieses zu bewerten ist, wer die Verantwortung trägt und welche Schritte notwendig sind, um das Problem $\mathrm{zu}$ lösen.

In Anlehnung an B. Scheufele (2003) und Entman (1993) werden Frames in der vorliegenden Arbeit als textliche Interpretationsrahmen verstanden, die bestimmte Problemdefinitionen, Ursachenzuschreibungen, explizite Bewertungen und Lösungszuschreibungen hervorheben und verknüpfen und auf diese Weise eine bestimmte Interpretation eines Themas nahelegen. Ausgehend von dieser Frame-Konzeption wird die (kognitive) Wirkung von Medien-Frames als zweistufiger Prozess verstanden: In einem ersten Schritt kann ein Medien-Frame durch den Rezipienten übernommen werden. In einem zweiten Schritt kann der gebildete Rezipienten-Frame bzw. das gebildete Muster kognitiver Vorstellungen die Einstellung des Rezipienten beeinflussen. 
Zur Unterscheidung von Kognition und Emotion hält LeDoux (1999) fest: „emotion and cognition are best thought of as separate but interacting mental functions mediated by separate but interacting brain systems" (S. 69). Entsprechend können kognitive und emotionale Prozesse unterschieden werden, wobei sich diese oft gegenseitig beeinflussen. Kognitive Prozesse sind unter anderem die Encodierung, Speicherung und das Abrufen von Informationen. Emotionale Reaktionen wie Ärger oder Traurigkeit resultieren, wenn ein Stimulus vom Individuum als relevant eingeschätzt wird (Way \& Masters, 1996). Emotionen werden somit als mentale Zustände verstanden, die aus kognitiven Evaluationsprozessen hervorgehen und mehrere Komponenten umfassen. Die affektive Komponente umfasst das subjektive Erleben des emotionalen Zustandes, die physiologische Komponente umfasst körperliche Reaktionen auf den Stimulus und die konative Komponente assoziierte Verhaltensintentionen und Verhaltensweisen (Oatley \& Jenkins, 1996). Emotionen beeinflussen ihrerseits kognitive Prozesse wie Wahrnehmung, Aufmerksamkeit und Erinnerung (Wirth \& Schramm, 2005). Obschon Kognition und Emotion unterschieden werden können, bestehen zwischen ihnen also ausgeprägte rekursive Beziehungen.

\section{Ansätze zu kognitiven Wirkungen von Medien-Frames}

Bisherige Ansätze erklären die Übernahme von Medien-Frames und ihre Wirkung auf Einstellungen durch kognitive Mechanismen. Die Erklärungen basieren meist auf einem Modell gedächtnisbasierter Meinungsbildung (Druckman \& Nelson, 2003; Matthes, 2007). Die Grundannahme ist, dass Individuen für die Urteilsbildung diejenigen Informationen verwenden, welche aus dem Langzeitgedächtnis abgerufen werden können. Gemäß dieser Perspektive, am prominentesten vertreten von Price und Tewksbury (1997), beeinflussen Frames, welche Kognitionen vom Rezipienten abgerufen werden können: Indem Frames bestimmte Themenaspekte hervorheben, erhöhen sie die Zugänglichkeit korrespondierender Kognitionen im Gedächtnis (vgl. auch Iyengar, 1991; B. Scheufele, 2003). Frames fungieren folglich als Medien-Primes (vgl. Peter, 2002), die beeinflussen, welche kognitiven Konzepte während der Urteilsbildung zur Verfügung stehen.

Da die Verknüpfung von Aspekten ein bestimmendes Merkmal von Medien-Frames ist, argumentieren Price und Tewksbury (1997), dass Frames vor allem Anwendbarkeitseffekte nach sich ziehen (vgl. auch D. Scheufele \& Tewksbury, 2007). Anwendbarkeitseffekte bezeichnen den Prozess, in welchem die Verknüpfung zweier Aspekte in einem Medien-Frame durch den Rezipienten übernommen wird (Price \& Tewksbury, 1997): Frames beeinflussen, welche Relationen zwischen Objekten vom Rezipienten als plausibel bzw. welche im Gedächtnis gespeicherten Vorstellungen als relevante Kriterien für die Urteilsbildung erachtet werden. Zum Beispiel kann ein Journalist Asylpolitik mit humanitären oder ökonomischen Erwägungen verknüpfen. Je nachdem welche Verknüpfung vorgenommen wird, dürfte der Rezipient eher humanitäre oder ökonomische Erwägungen als angemessene Bewertungsgrundlage erachten.

Nelson und Kollegen (1997) argumentieren ähnlich wie Price und Tewksbury (1997), dass Medien-Frames ihre Wirkung über die Verknüpfung von Konzepten entfalten. Sie nehmen an, dass weniger die Verfügbarkeit eines kognitiven Konzeptes für dessen Verwendung ausschlaggebend ist als vielmehr die Wichtigkeit, die dem Konzept vom Rezipienten zugeschrieben wird. Medien-Frames beeinflussen demnach in erster Linie, welche Erwägungen vom Rezipienten als wichtig eingeschätzt werden. Wie Chong und Druckman (2007) erläutern, impliziert diese Perspektive, dass Medien-Frames nicht so 
sehr automatische und unbewusste Entscheidungsprozesse anstoßen als vielmehr bewusste Deliberationsprozesse.

Die genannten Ansätze zeichnen ein Bild kognitiver Framing-Effekte: MedienFrames beeinflussen, welche Informationen im Gedächtnis zugänglich sind und für die Urteilsbildung anwendbar bzw. relevant erscheinen. Im weitesten Sinn können Wirkungen von Medien-Frames deshalb als kognitive Lernprozesse verstanden werden, die zur Übernahme einer bestimmten Perspektive durch den Rezipienten führen. Die Ansätze implizieren auch, dass Framing-Effekte das Resultat unterschiedlich elaborierter Informationsverarbeitungsprozesse sind. Zugänglichkeitseffekte dürften vor allem vorkommen, wenn eine oberflächliche Verarbeitung vorliegt und Urteile auf den gerade verfügbaren Informationen basieren. Sind Rezipienten hingegen ausreichend motiviert und besitzen die notwendigen kognitiven Ressourcen, so können sie eine größere Menge von Informationen berücksichtigen und diese Informationen bezüglich ihrer Stringenz und Eignung als Entscheidungsgrundlage beurteilen. Bei einer intensiven Informationsverarbeitung dürften deshalb Anwendbarkeits- und Wichtigkeitseffekte bedeutender sein (vgl. Chong \& Druckman, 2007; Nelson et al., 1997).

\section{Empirische Befunde zu emotionalen Wirkungen von Medien-Frames}

In den erörterten Erklärungsansätzen für Framing-Effekte werden Emotionen nicht berücksichtigt. Allerdings existieren mittlerweile erste Studien, die emotionale Prozesse bei der Rezeption von Medien-Frames untersuchen. Diese Studien zeigen, dass MedienFrames Emotionen auslösen können und dass diese Emotionen Einstellungen beeinflussen.

Mehrere Studien belegen, dass Ursachenzuschreibungen in Zeitungsartikeln beeinflussen, welche Emotionen ausgelöst werden. Nerb (2000) zeigte mittels Experimenten, dass Rezipienten mehr Ärger empfinden, wenn in einem Artikel eine Verantwortungszuschreibung vorgenommen wird: Wurde ein Akteur für ein Unglück verantwortlich gemacht, dann wurde mehr Ärger ausgelöst, als wenn eine situative Ursachenzuschreibung vorlag. Weiterhin wurde mehr Ärger und weniger Traurigkeit ausgelöst, wenn ein Akteur ein Unglück wissentlich verursachte, als wenn er die Konsequenzen seines Handelns nicht abschätzen konnte. Obschon Nerb nur am Rande auf Framing-Ansätze Bezug nimmt, deuten die Ergebnisse darauf hin, dass Frame-Komponenten wie die Ursachenzuschreibung beeinflussen können, welche Emotionen ausgelöst werden.

Entsprechende Befunde stammen von Kim und Cameron (2011). In einem Experiment wurde den Probanden ein Artikel gezeigt, der über einen Explosionsunfall mit einem Telefonakku berichtete. Jeweils der Hälfte der Probanden wurde eine von zwei Frame-Versionen präsentiert. Der erste Frame sollte Ärger erzeugen, indem das bewusste Fehlverhalten des Akku-Herstellers fokussiert wurde. Der zweite Frame sollte Traurigkeit erzeugen, indem die Folgen für die Opfer fokussiert wurden. Wie erwartet produzierten die beiden Frames unterschiedlich viel Ärger und Traurigkeit. Ebenso konnten Kühne und Schemer (2013) in einer Experimentalstudie zum Thema Verkehrssicherheit belegen, dass ein Frame, der das fahrlässige Verhalten von Autofahrern fokussierte, mehr Ärger auslöste als ein Frame, welcher die Folgen für Opfer fokussierte.

Weitere Studien untersuchten die Auslösung von Emotionen durch Gewinn- und Verlust-Frames. Cho und Boster (2008) stellten fest, dass ein Gewinn-Frame einer Werbung für Drogenprävention mehr Freude und Zufriedenheit auslöste, während ein Verlust-Frame mehr Ärger, Angst und Traurigkeit auslöste. Ebenso zeigten Shen und Dillard (2007), dass Gewinn-Frames mehr positive Emotionen auslösen und VerlustFrames mehr negative Emotionen. 
In mehreren Studien wurde die Emotionsauslösung durch episodische und thematische Frames untersucht. Gross (2008) zeigte Probanden einen Artikel gegen obligatorische Minimalstrafen bei Drogendelikten. Sie stellte fest, dass episodische Frames, welche das Einzelschicksal einer Angeklagten fokussierten, unter anderem mehr Sympathie und Mitleid auslösten als ein thematischer Frame, welcher Details über die Richtlinie zur Verurteilung von Tätern in den Mittelpunkt stellte. Zudem zeigten Gross und Kollegen, dass die Emotionsauslösung durch Frames durch Merkmale des Rezipienten moderiert wird, wie zum Beispiel die Voreinstellung zu einem Thema (Gross \& Brewer, 2007) oder die politische Ideologie (Gross \& D’Ambrosio, 2004).

Emotionale Wirkungen von Medien-Frames auf Einstellungen wurden bislang in wenigen Studien untersucht. In der bereits erwähnten Studie untersuchte Gross (2008), welche Folgen durch Frames induzierte Emotionen für die Einstellung gegenüber obligatorischen Minimalstrafen bei Drogenverbrechen haben. Sie stellte fest, dass die episodischen Frames im Vergleich zum thematischen Frame die Ablehnung von Minimalstrafen über die Auslösung von Sympathie für Drogenabhängige erhöhten. Das heißt, der Framing-Effekt auf die Einstellung wurde durch die ausgelöste Sympathie mediiert. Ähnlich zeigen Kim und Cameron (2011), dass der Artikel zu einem Explosionsunfall mit einem Telefonakku eine signifikant negativere Einstellung gegenüber der Herstellerfirma erzeugte, wenn ein Ärger-Frame anstelle eines Traurigkeits-Frames verwendet wurde. Offenbar trübte der ausgelöste Ärger die Bewertung des Unternehmens. Schließlich stellten Kühne und Schemer (2013) in ihrer Studie fest, dass der Ärger-Frame im Vergleich zum Traurigkeits-Frame dazu führte, dass Strafmaßnahmen stärker befürwortet wurden und Individuen eher bereit waren, sich für die Bestrafung von Verkehrssündern politisch zu engagieren.

Die empirischen Befunde deuten darauf hin, dass Medien-Frames neben kognitiven Prozessen auch emotionale Reaktionen auslösen können, welche die Einstellung der Rezipienten beeinflussen. Ein umfassendes Modell für die Erklärung emotionaler Framing-Effekte wurde bislang aber nicht formuliert. Im Folgenden wird auf Basis von Emotionstheorien und Ansätzen zu kognitiven Framing-Effekten ein solches Modell entwickelt. Dieses muss drei Fragen beantworten. Als kommunikationswissenschaftliches Modell muss es in erster Linie erklären, wie spezifische Medien-Frames bestimmte Emotionen auslösen. Zweitens muss es erläutern, was für Wirkungen durch Frames induzierte Emotionen auf den Rezipienten haben können. Drittens muss erörtert werden, von welchen Randbedingungen emotionale Wirkungen abhängig sind. Im Folgenden werden diese Fragen beantwortet.

\section{Die Emotionsauslösung durch Medien-Frames}

In diesem Abschnitt wird zuerst erörtert, wie mittels emotionspsychologischer Einschätzungstheorien die Auslösung von Emotionen durch Medien-Frames erklärt werden kann. Anschließend wird erläutert, dass die spezifische emotionale Reaktion davon abhängig ist, wie ein Medien-Frame aufgebaut ist. Schließlich werden exemplarisch zwei Typen von Frames diskutiert, die Ärger bzw. Traurigkeit auslösen sollten.

\subsection{Die kognitive Einschätzung von Medien-Frames und Auslösung von Emotionen}

Die Auslösung von Emotionen durch Nachrichten wird meist mittels einschätzungstheoretischer Ansätze der Emotionspsychologie erklärt (z. B. Nerb, 2000). Einschätzungstheorien basieren auf der Grundidee, dass Emotionen wie Ärger oder Traurigkeit aus kognitiven Einschätzungen bzw. Interpretationen von Ereignissen und Situationen 
hervorgehen (Lazarus, 1991; Ortony, Clore \& Collins, 1988; Scherer, 2001). In Übereinstimmung mit den im zweiten Abschnitt vorgenommenen begrifflichen Präzisierungen von Kognition und Emotion postulieren diese Ansätze also einen inhärenten $\mathrm{Zu}$ sammenhang zwischen Kognition und Emotion. Angenommen wird, dass Individuen kontinuierlich ihre Umgebung evaluieren und dabei eine Reihe charakteristischer Situationsbewertungen vornehmen. Die kognitiven Bewertungen laufen dabei oft automatisch und unbewusst ab, können aber auch das Ergebnis eines elaborierten Evaluationsprozesses sein (z. B. Leventhal \& Scherer, 1987). Je nach Autor werden in diesem Prozess unterschiedliche Einschätzungskriterien als zentral erachtet. Häufig genannt werden die (positive oder negative) Valenz des Ereignisses, die Sicherheit des Ereignisses, die personale Kontrollierbarkeit über sowie Verantwortung für das Ereignis (z. B. Ortony et al., 1988; Scherer, 2001). Spezifische Emotionen gehen aus bestimmten Mustern bzw. Konfigurationen von kognitiven Einschätzungen hervor. Wird ein Ereignis zum Beispiel als stark negativ und kontrollierbar eingeschätzt sowie die Verantwortung einem Akteur zugeschrieben, dann sollte Ärger resultieren. Die Bedeutung einer Einschätzungskonfiguration für das Individuum wird von Lazarus (1991) core relational theme genannt. Bei Ärger ist das Kernthema zum Beispiel, dass jemand dem Individuum ungerechtfertigt Schaden zufügt. Emotionen sind somit das Resultat subjektiver Bewertungsprozesse, so dass dieselbe Situation von verschiedenen Individuen unterschiedlich eingeschätzt werden kann und unterschiedliche Emotionen hervorrufen kann.

Aus Einschätzungstheorien kann für die Wirkung von Medien-Frames folgende Hypothese abgeleitet werden: Über die Hervorhebung und Verknüpfung bestimmter Aspekte eines Themas können Medien-Frames Interpretationen bzw. kognitive Einschätzungen nahelegen, die Emotionen auslösen. Stimmt die central organizing idea (Gamson \& Modigliani, 1987) eines Artikels also mit dem core relational theme (Lazarus, 1991) einer spezifischen Emotion überein, dann sollte die entsprechende Emotion ausgelöst werden. Die etablierten kognitiven Zugänglichkeits- und Anwendbarkeitseffekte können somit Emotionen auslösen, wenn sie zur Aktivierung emotionsspezifischer Einschätzungsmuster führen. Einschätzungsmuster können dabei durch elaborierte Interpretationsprozesse bzw. Anwendbarkeitseffekte hervorgebracht oder durch automatische Zugänglichkeitseffekte aktiviert werden (vgl. Leventhal \& Scherer, 1987). Die Emotionsauslösung infolge Zugänglichkeits- und Anwendbarkeitseffekten muss sich dabei nicht gegenseitig ausschließen. In Abhängigkeit von der Intensität der Informationsverarbeitung dürften aber eher Zugänglichkeits- oder Anwendbarkeitseffekte für die Emotionsauslösung verantwortlich sein.

\subsection{Der Zusammenhang zwischen Frame-Komponenten und Einschätzungsmustern}

Andere Autoren haben bereits Einschätzungstheorien für die Erklärung der Emotionsauslösung durch Medien-Frames herangezogen und ähnliche Hypothesen formuliert (Gross, 2008; Kim \& Cameron, 2011; Nabi, 2003). In den bisherigen theoretischen Erörterungen ist die Integration von Framing- und Emotionstheorien allerdings unzureichend. Das Argument, dass Medien-Frames kognitive Einschätzungen beeinflussen können, ist für die Erklärung emotionaler Framing-Effekte zu unspezifisch, da es nicht berücksichtigt, aus welchen Komponenten sich Medien-Frames zusammensetzen und welche kognitiven Einschätzungen durch diese Komponenten beeinflusst werden. Wird der Frame-Begriff aber ernst genommen, das heißt, wird der Begriff nicht bloß als Metapher, sondern als analytische Definition eines empirisch beobachtbaren Phänomens verstanden, dann muss ein Modell emotionaler Framing-Effekte die - entsprechend dem vorliegenden Frame-Verständnis - zentralen Frame-Komponenten explizit mit den zen- 
tralen emotionstheoretischen Einschätzungskriterien in Beziehung setzen. Diese theoretische Präzisierung ist notwendig, um Hypothesen über emotionale Wirkungen spezifischer Medien-Frames zu formulieren. Das Vorgehen setzt eine klare Definition voraus, aus welchen Komponenten sich ein Medien-Frame zusammensetzt. Wir folgen der Definition von Entman (1993), die vier Frame-Komponenten identifiziert (Problemdefinition, Ursachenzuschreibung, explizite Bewertung und Lösungszuschreibung). Analytisch verstehen wir diese Komponenten sowie die zentralen Einschätzungskriterien als Variablen, die verschiedene Ausprägungen annehmen können. Wir postulieren folglich, dass bestimmte Ausprägungen der Frame-Komponenten mit bestimmten Ausprägungen der Einschätzungskriterien korrespondieren: Medien-Frames bestehen aus vier Komponenten, welche die kognitiven Einschätzungen der Rezipienten beeinflussen. Die Problemdefinition dürfte in erster Linie bestimmen, welcher Aspekt eines Problems von Rezipienten als zentral eingeschätzt wird. Die Ursachenzuschreibung dürfte vor allem die Einschätzung der Verantwortlichkeit beeinflussen, die explizite Bewertung die Einschätzung der Valenz und die Lösungszuschreibung die Einschätzung der Kontrollierbarkeit. Bei der Aktivierung emotionsspezifischer Einschätzungsmuster werden Emotionen ausgelöst. Hervorzuheben ist, dass die Komponenten eines Medien-Frames die Einschätzungen der Rezipienten nicht determinieren. Da kognitive Einschätzungen von Merkmalen des Kontextes und des Individuums beeinflusst werden (Lazarus, 1991; Scherer, 2001), kann ein und derselbe Medien-Frame zu unterschiedlichen Evaluationen führen. Medien-Frames legen somit eine Sichtweise nahe, diese muss aber nicht zwingend vom Rezipienten übernommen werden (Chong \& Druckmann, 2007). Im Folgenden werden die vier Frame-Komponenten nach Entman (1993) beschrieben. Danach wird exemplarisch erörtert, wie idealtypische Frames ausgestaltet sein müssen, um Ärger und Traurigkeit auszulösen.

Die Problemdefinition verstehen wir in Anlehnung an Matthes (2007) als einen Blickwinkel auf ein Thema: „Mit der Problemdefinition wird festgelegt, warum ein Thema wichtig ist und öffentlich diskutiert wird. Die Problemdefinition ,spannt' gewissermaßen den Rahmen auf und definiert, worüber gesprochen wird und welche Akteure relevant sind“ (S. 134-135). Zentrale Aspekte, über die gesprochen werden kann, sind Ereignisse, Akteure und Betroffene. Das heißt: Die Strukturierungsleistung der Problemdefinition besteht in erster Linie in der Wahl der relevanten Ereignisse, Akteure und Betroffenen, die ein Thema ausmachen. Neben dieser sachlichen Eingrenzung wird auch eine zeitliche Verortung des Problems vorgenommen (d. h.: Handelt es sich um ein früheres, ein aktuelles oder ein zukünftiges Problem?).

Die Ursachenzuschreibung nennt die Ursachen für ein gesellschaftliches Problem. Unterschieden werden situative und personale Ursachenzuschreibungen (z. B. Iyengar, 1991). Probleme mit situativen Ursachen sind nicht auf das Handeln eines individuellen oder kollektiven Akteurs zurückzuführen. Vielmehr sind äußere Umstände verantwortlich. Zum Beispiel stellt ein Unwetter eine situative Ursache für eine Überschwemmung dar. Kann ein Problem auf das Handeln eines Akteurs zurückgeführt werden, dann liegt eine personale Verantwortung vor. Mangelnde Vorkehrungen der Regierung zum Unwetterschutz stellen eine personale Ursache für eine Überschwemmung dar.

Drittens werden in Medien-Frames explizite Bewertungen vorgenommen. Das Problem selbst oder Aspekte des Problems, wie Ereignisse oder relevante Akteure (Entman, 1993), werden auf einem Kontinuum zwischen „sehr negativ“ und „sehr positiv“ verortet. Bewertungen inhärent negativer Problemstellungen, wie Arbeitslosigkeit, Kriminalität oder Umweltverschmutzung, können sich bezüglich des Grades ihrer Negativität unterscheiden. Die explizite Bewertung stellt also eine Beurteilung dar, wie schwerwie- 
gend und dringend ein Problem ist. Ähnlich kann die explizite Bewertung verdeutlichen, wie wünschenswert bzw. problematisch das Verhalten eines Akteurs ist.

Die Lösungszuschreibung gibt schließlich an, ob es Akteure gibt, die im Stande sind, das Problem zu lösen, und wer diese Akteure sind. Akteuren kann die Lösungskompetenz auch explizit abgesprochen werden. Grundsätzlich kann also auch bestritten werden, dass überhaupt ein Akteur die Fähigkeit besitzt, das Problem zu lösen. Neben der Identifikation der Akteure werden auch Handlungsaufforderungen ausgesprochen. Das heißt, einflussreiche Akteure werden aufgefordert, unterstützende Maßnahmen zu vollziehen und der Problemlösung abträgliche Maßnahmen zu unterlassen.

\section{3 Ärger-und Traurigkeits-Frames}

Ausgehend von Entmans (1993) Frame-Verständnis wird nun erörtert, wie idealtypische Ärger- und Traurigkeits-Frames aufgebaut sind. Grundsätzlich könnten auch idealtypische Frame-Konfigurationen für andere Emotionen wie Hoffnung, Angst oder Stolz definiert werden.

Ärger stellt eine negative Emotion dar, die ausgelöst wird, wenn ein Individuum durch ein Hindernis vom Erreichen seiner Ziele abgehalten wird oder von jemandem absichtlich geschädigt wird, das Individuum zugleich aber die Fähigkeit besitzt, die Situation zu bewältigen bzw. den Schuldigen zu bestrafen. In der Terminologie der Einschätzungstheorien resultiert Ärger aus der Einschätzung, dass ein Sachverhalt negativ und kontrollierbar ist, eine hohe Eintretenswahrscheinlichkeit besitzt und die Verantwortung dafür von einem Akteur getragen wird. Ärger leitet das Individuum an, ein Problem aktiv anzugehen bzw. den Verursacher anzugreifen (Lazarus, 1991).

Die Problemdefinition eines Ärger-Frames fokussiert das Verhalten von Akteuren (vgl. Ortony et al., 1988). Im Mittelpunkt stehen Verhaltensweisen, die gesellschaftlich unerwünscht sind, da sie eine bestimmte Norm verletzten oder mit negativen Konsequenzen verbunden sind. Meist fokussiert ein Ärger-Frame unerwünschte Verhaltensweisen, die bereits vollzogen wurden, ist also in die Vergangenheit gerichtet. Ärger kann aber auch ausgelöst werden, wenn ein Akteur eine unerwünschte Verhaltensweise in Zukunft mit hoher Sicherheit ausüben wird.

In einem Ärger-Frame wird die Verantwortung für ein gesellschaftliches Problem einem Akteur zugeschrieben. Je größer der Beitrag eines Akteurs zur Verursachung eines Problems ist, desto größer ist seine Verantwortung. Selbst bei personaler Verursachung eines Problems kann die Verantwortlichkeit allerdings begrenzt sein und Ärger abgeschwächt werden. Die Verantwortung wird reduziert, wenn der Akteur nicht absichtlich gehandelt hat, ein übergeordnetes normatives Ziel verfolgt wurde, oder die Konsequenzen des Handelns nicht abgeschätzt werden konnten (Nerb, 2000; Ortony et al., 1988).

Explizit negative Bewertungen innerhalb eines Medien-Frames begünstigen die Auslösung von Ärger. Die Wahrnehmung des Rezipienten, dass ein negativer Sachverhalt vorliegt, ist Voraussetzung dafür, dass eine Verantwortungszuschreibung Ärger auslöst. Die explizite Bewertung eines gesellschaftlichen Problems unterstützt folglich die Entstehung von Ärger. Zudem wird Ärger begünstigt, wenn das Verhalten eines Akteurs explizit als verwerflich bzw. Normbruch dargestellt wird (vgl. Ortony et al., 1988).

Schließlich wird die Auslösung von Ärger begünstigt, wenn ein Medien-Frame ein Problem als lösbar bzw. kontrollierbar darstellt. Ein Problem kann als kontrollierbar dargestellt werden, indem es im Text explizit als lösbar bezeichnet wird und/oder Akteure genannt werden, die das Problem lösen können, sowie Handlungsempfehlungen ausgesprochen werden. Kontrollierbarkeit impliziert, dass einerseits der Verursacher für sein Verhalten sanktioniert werden kann und andererseits die negativen Folgen des Ver- 
haltens korrigiert bzw. abgewendet werden können. Ärger resultiert demnach, wenn ein Medien-Frame darauf hindeutet, dass die Bestrafung des Verursachers und die aktive Problembewältigung möglich sind. Deshalb kann auch die explizite Empfehlung von Maßnahmen, die auf die Bestrafung des Verantwortlichen abzielen, die Auslösung von Ärger unterstützen.

Ein idealtypischer Ärger-Frame fokussiert also die unerwünschten Handlungen von Akteuren, die für ein Problem verantwortlich gemacht werden. Das Verhalten der Akteure wird explizit kritisiert und es wird empfohlen, die Verantwortlichen zu bestrafen. Ein Beispiel für einen Ärger-Frame stellt das Berichterstattungsmuster zur Plagiatsaffäre Guttenberg dar: Fokussiert wurde das Verhalten von Guttenberg, dem die Verantwortung für die Verfassung eines Plagiats zugeschrieben wurde. Das Verhalten wurde explizit als Normbruch bewertet und als Lösung für das Problem der Rücktritt von Guttenberg vorgeschlagen.

Traurigkeit ist eine negative Emotion, die ausgelöst wird, wenn jemand einen schwerwiegenden Verlust erleidet, der kaum kompensiert werden kann. Im weiteren Sinn stellt Traurigkeit eine Reaktion auf unerwünschte Ereignisse dar, die mit gravierenden negativen Implikationen verbunden sind (Schmitt \& Mees, 2000). In der Terminologie der Einschätzungstheorien resultiert Traurigkeit aus der Einschätzung, dass ein Sachverhalt negativ und kaum kontrollierbar ist, eine hohe Eintretenswahrscheinlichkeit besitzt und dass keine personale Verantwortung vorliegt. Traurigkeit geht mit der Handlungstendenz einher, den erlittenen Verlust zu kompensieren und/oder Trost zu suchen (Lazarus, 1991; Ortony et al., 1988). Traurigkeit leitet das Individuum an, Opfern zu helfen und entstandene Schäden zu reparieren (Nerb, 2000).

Die Problemdefinition eines Traurigkeits-Frames fokussiert die negativen Konsequenzen für Betroffene, mit denen ein Problem einhergeht (vgl. Ortony et al., 1988). Im Mittelpunkt stehen die Schwierigkeiten und das Leiden von Individuen. Anstatt die Verhaltensweisen von Akteuren zu fokussieren, werden also die Implikationen für Betroffene bzw. Opfer akzentuiert. In Übereinstimmung mit Ärger-Frames fokussieren Traurigkeits-Frames gegenwärtige negative Situationen. Allerdings kann Traurigkeit auch ausgelöst werden, wenn zukünftige Verluste mit hoher Sicherheit eintreten.

In Traurigkeits-Frames werden situative Ursachenzuschreibungen vorgenommen. Es wird argumentiert, dass das Problem nicht kontrolliert werden konnte und folglich kein Akteur die Verantwortung trägt. Insbesondere wird argumentiert, dass die Betroffenen bzw. Opfer ohne eigenes Verschulden in die Unglückslage geraten sind. Das heißt, den Betroffenen wird die Verantwortung abgesprochen. Selbst wenn ein spezifischer Akteur ein Problem verursacht hat, kann die Verantwortungszuschreibung abgeschwächt sein, wenn erörtert wird, dass der Akteur nicht intentional handelte, ein höheres normatives Ziel verfolgte oder die Konsequenzen seines Tuns nicht abschätzen konnte (Nerb, 2000).

Der ideale Traurigkeits-Frame umfasst eine negative Bewertung der Situation der Betroffenen bzw. der Opfer. Beteiligte Akteure und Betroffene werden normalerweise nicht negativ evaluiert. Vielmehr werden deren Verhaltensweisen explizit als positiv bewertet. In Übereinstimmung mit der situativen Ursachenzuschreibung deutet die explizit positive Bewertung der Verhaltensweisen darauf hin, dass die missliche Lage eintrat, obwohl sich alle Beteiligten korrekt verhielten.

Schließlich wird in Traurigkeits-Frames angezeigt, dass die Problemlösung schwierig oder sogar unmöglich ist. Es wird entsprechend argumentiert, dass kein Akteur die Fähigkeit besitzt, das Problem zu lösen, oder dass ein erheblicher Aufwand betrieben werden muss. Wenn eine Lösung des Problems denkbar ist, dann fokussieren Handlungs- 
aufforderungen Maßnahmen, um die Betroffenen bzw. Opfer zu unterstützen und deren Leid zu verringern.

Ein idealer Traurigkeits-Frame fokussiert also die negativen Konsequenzen für Betroffene. Situative Ursachen werden für das Problem verantwortlich gemacht und spezifischen Akteuren wird die Verantwortung abgesprochen. Handlungsaufforderungen fokussieren Maßnahmen zur Opferhilfe. Ein Beispiel für einen Traurigkeits-Frame stellt die Berichterstattung zur Tsunami-Katastrophe in Japan dar. Fokussiert wurden die Zerstörung der Infrastruktur sowie die Opfer der Flutkatastrophe. Spezifischen Akteuren wurde zunächst kaum Verantwortung zugeschrieben und es wurde empfohlen, die japanische Regierung bei der Bewältigung der Katastrophe zu unterstützen.

\section{Die Wirkung durch Frames ausgelöster Emotionen auf den Rezipienten}

Zahlreiche psychologische Studien beschäftigen sich mit Einflüssen emotionaler Zustände auf die Urteilsbildung (vgl. Forgas, 1995; Lerner \& Keltner, 2000). Ein häufig referiertes Modell zur Erklärung der Wirkung von Emotionen ist das Appraisal Tendency Framework (Han, Lerner \& Keltner, 2007; Lerner \& Keltner, 2000), welches Überlegungen aus kognitiven Einschätzungstheorien und funktionalen Emotionstheorien integriert. Von Einschätzungstheorien übernimmt der Ansatz die Idee, dass Emotionen aus kognitiven Einschätzungsmustern hervorgehen. Dieser erste Schritt im Wirkungsprozess wird „cognition-to-emotion“ genannt. Zentral ist die im ATF vorgenommene Erweiterung, dass emotionale Reaktionen in einem zweiten Schritt („emotion-to-cognition“) kognitive Bewertungen beeinflussen: „[...] emotions not only can arise from but give rise to an implicit cognitive predisposition to appraise future events in line with central appraisal patterns or appraisal themes that characterize the emotions" (Han et al., 2007: 160). Das heißt, der Bedeutungsgehalt von Emotionen fließt in die Informationsverarbeitung und Urteilsbildung ein. Diese emotionale Rückkopplung wird durch funktionale Emotionstheorien (Lazarus, 1991; Ortony et al., 1988) begründet, laut denen Emotionen die Anpassung des Individuums an seine Umwelt erleichtern, indem sie verschiedene Subsysteme des Individuums koordinieren (u. a. das motivationale und das verhaltensbezogene System; vgl. Scherer, 2001). Da jede Emotion aus einer spezifischen Einschätzungskonfiguration hervorgeht und für das Individuum eine spezifische Bedeutung besitzt, führt laut dem ATF jede Emotion zu einer spezifischen Informationsverarbeitung und Urteilsbildung. Zum Beispiel fördert Ärger individuelle Schuldzuschreibungen, während Traurigkeit situative Attributionen fördert. Zwischen Kognitionen und Emotionen besteht folglich eine rekursive Beziehung (vgl. auch Abschnitt 2): Kognitive Einschätzungen lösen Emotionen aus und Emotionen beeinflussen wiederum kognitive Einschätzungen. Da sich Einstellungen zu einem bedeutenden Teil aus kognitiven Erwägungen speisen (Eagly \& Chaiken, 1993), haben die erörterten kognitiven Wirkungen Implikationen für die Meinungsbildung: Emotionskongruente Kognitionen fördern emotionskongruente Einstellungen.

Weitere Ansätze postulieren, dass Emotionen als Urteilsheuristiken verwendet werden können (Forgas, 1995). Demnach können Emotionen auf Einstellungsobjekte projiziert werden und dadurch die Bewertung des Objekts beeinflussen. Wird aktuell erlebter Ärger zum Beispiel auf einen Politiker projiziert, dann dürfte dies eine ablehnende Haltung unterstützen. Hervorzuheben ist, dass durch Emotionen geformte Einstellungen oft eine andere Qualität besitzen als kognitiv gebildete Einstellungen. Emotionen führen unter anderem zu stärkeren und extremeren Einstellungen und erhöhen die Zugänglichkeit von Einstellungen (vgl. Eagly \& Chaiken, 1993). Selbst wenn die kognitive 
und die emotionale Verarbeitung den selben Standpunkt nahelegen, sind also unterschiedliche Urteile zu erwarten.

Eine wesentliche Wirkung von Emotionen stellt schließlich ihr Einfluss auf Motivation und Verhalten dar. Laut der funktionalen Perspektive unterstützen Emotionen die Adaption von Individuen, indem sie bestimmte Verhaltensweisen initiieren, die angesichts der situativen Anforderungen erforderlich sind (Lazarus, 1991). Jede Emotion ist demnach mit einem spezifischen Handlungsziel verbunden. So leitet Ärger das Individuum zum Beispiel an, den Auslöser des Ärgers zu bestrafen, während Traurigkeit die Unterstützung Betroffener veranlasst. Insgesamt bedeutet dies für die Konzeptualisierung von Framing-Effekten, dass bestimmte Berichterstattungsmuster Emotionen induzieren können, die zu emotionskongruenten Einstellungen und Verhaltensintentionen führen.

\section{Randbedingungen emotionaler Wirkungen von Medien-Frames}

Die Framing-Forschung hat mittlerweile verschiedene Randbedingungen bzw. Moderatoren kognitiver Wirkungen von Medien-Frames bestimmt, wie zum Beispiel das Vorwissen und die Voreinstellung der Rezipienten (z. B. Druckman \& Nelson, 2003). Ein Modell emotionaler Framing-Effekte muss neben diesen Moderatoren auch Variablen berücksichtigen, die einen Einfluss auf emotionale Prozesse ausüben. Theoretische Erwägungen und erste empirische Befunde deuten darauf hin, dass emotionale FramingEffekte durch spezifische Moderatoren beeinflusst werden. $\mathrm{Zu}$ unterscheiden sind Variablen, welche die Emotionsauslösung moderieren, und Variablen, welche den Einfluss von Emotionen auf Einstellungen moderieren.

Wie stark die Emotionen sind, die das Framing eines Nachrichtenbeitrags auslöst, hängt zum einen von Merkmalen des Beitrags wie der thematischen Relevanz, der narrativen Struktur und dem Realitätsgrad der Darstellung ab. Beiträge zu Themen, die eine größere Relevanz besitzen bzw. mit größeren (potenziellen) Implikationen für das Individuum verbunden sind, sollten stärkere Emotionen auslösen (Sonnemans \& Frijda, 1995). Ebenso unterstützt eine narrative Darstellung eines Themas emotionale Reaktionen. Medien-Frames, welche Ereignisse und Handlungen von Personen anstelle von abstrakten Informationen fokussieren, sollten demnach stärkere Emotionen hervorrufen (Gross, 2008; Zillmann \& Knobloch, 2001). Laut Ortony et al. (1988) steigt die Intensität von Emotionen mit dem Grad, zu welchem eine Situation von einer Person als real wahrgenommen wird. Mediale Darstellungen mit einem hohen Realitätsgrad sollten demnach intensivere Emotionen hervorrufen.

Neben Merkmalen des Stimulus beeinflussen Merkmale des Rezipienten die Emotionsauslösung. Gewisse Individuen neigen eher dazu, auf Situationen mit bestimmten Emotionen wie Ärger oder Traurigkeit zu reagieren (Lerner \& Keltner, 2000). Menschen unterscheiden sich auch bezüglich ihrer Neigung, emotionale Erfahrungen zu suchen. Ein hohes Bedürfnis nach emotionalen Erlebnissen unterstützt intensive emotionale Reaktionen (Maio \& Esses, 2001). Die Emotionsauslösung bei der eingehenden Rezeption eines Nachrichtenbeitrags bedingt auch, dass der Inhalt des Beitrags mehr oder weniger verstanden wird. Politische Kompetenz unterstützt zum Beispiel das Verstehen politischer Informationen und somit emotionale Reaktionen auf politische Beiträge (Miller, 2011). Ähnlich dürfte die Emotionsauslösung bei einer oberflächlichen Informationsverarbeitung dadurch beeinflusst werden, ob emotionale Schemata vorliegen, die aktiviert werden können (vgl. Leventhal \& Scherer, 1987). Thematische Voreinstellungen und Weltanschauungen sind weitere Moderatoren der Emotionsauslösung. Je nachdem, ob die Präferenzen eines Rezipienten in einem Nachrichtenbeitrag angegriffen oder 
unterstützt werden, können mehr oder weniger starke positive bzw. negative Emotionen ausgelöst werden (Gross \& D’Ambrosio, 2004; Gross \& Brewer, 2007).

Weitere Moderatoren beeinflussen, wie stark emotionale Einflüsse auf Einstellungen sind. Grundsätzlich unterbinden feste Voreinstellungen Einstellungsänderungen (Druckman \& Nelson, 2003) und somit emotionale Framing-Effekte. Emotionseinflüsse können auch vermindert werden, wenn das Individuum eine Emotionsregulation vornimmt, das heißt, wenn es versucht, seine Emotionen abzuschwächen. Emotionsregulation wird vor allem von Individuen vorgenommen, die bestimmte emotionale Reaktionen als besonders unangenehm erleben oder aus normativen Erwägungen ablehnen (Harmon-Jones et al., 2011). Ähnlich sollten emotionale Framing-Effekte für Individuen stärker ausfallen, die eine stärkere Neigung haben, sich bei Entscheidungen an ihren eigenen Gefühlen zu orientieren (Maio \& Esses, 2001). Bei einer elaborierten Informationsverarbeitung fungiert die Neigung, sich intensiv mit Informationen auseinanderzusetzen, als Moderator von Emotionseinflüssen: Je intensiver über ein Thema nachgedacht wird, desto eher können Emotionen die Einstellungsbildung beeinflussen - ein Effekt, der affektives Priming genannt wird (Forgas, 1995). Bei einer oberflächlichen Verarbeitung fallen Emotionseinflüsse auf Einstellungen indessen stärker aus, wenn beim Rezipienten Wissensstrukturen vorliegen, die durch Emotionen aktiviert werden können (Nabi, 2003).

Die referierten Studien deuten darauf hin, dass emotionale Framing-Effekte vielfach von anderen Randbedingungen abhängig sind als kognitive Effekte. Dabei können bestimmte Variablen unterschiedliche Implikationen für die Auslösung und die Wirkung von Emotionen haben. Zum Beispiel dürften starke politische Überzeugungen die Emotionsauslösung begünstigen, Einstellungsänderungen infolge der emotionalen Reaktion aber eher unterbinden. Da es sich bei den referierten Studien vorwiegend um keine Framing-Studien handelt, ist der moderierende Einfluss der erörterten Variablen auf emotionale Framing-Prozesse empirisch noch zu überprüfen.

\section{Ein Modell emotionaler Wirkungen von Medien-Frames}

Die bisherige Framing-Forschung fokussierte in erster Linie kognitive Wirkungen von Medien-Frames. Im Beitrag wurden zentrale Aspekte eines integrativen Wirkungsmodells skizziert, das beschreibt, wie kognitive Verarbeitungsprozesse emotionale Reaktionen hervorbringen können, welche in die Urteilsbildung einfließen. Das Modell basiert auf zwei zentralen Postulaten: Erstens wird argumentiert, dass Medien-Frames Konfigurationen mehrerer Komponenten darstellen (der Problemdefinition, Ursachenzuschreibungen, expliziten Bewertungen und Lösungszuschreibungen). Diese FrameKomponenten beeinflussen die kognitiven Einschätzungen der Rezipienten, indem sie eine bestimmte Sichtweise auf das Thema des Beitrages nahelegen. Werden bestimmte Einschätzungsmuster generiert, so resultieren spezifische Emotionen. Zweitens fördern durch Medien-Frames induzierte Emotionen emotionskongruente Einstellungen und Verhaltensweisen. Aus der Integration der beiden Postulate folgt, dass emotionale Framing-Effekte als ein dreistufiger Prozess zu verstehen sind: Erstens aktivieren Medien-Frames kognitive Einschätzungen. Bestimmte Muster kognitiver Einschätzungen können zweitens Emotionen auslösen. Diese führen schließlich zu emotionskongruenten Einstellungen und Verhaltensweisen. Schließlich wird postuliert, dass die Auslösung und Wirkung von Emotionen - analog zu kognitiven Zugänglichkeits- und Anwendbarkeitsprozessen - von spezifischen Moderatoren abhängig sind. Das Modell ist in der Abbildung zusammengefasst. 
Abbildung: Schematische Darstellung der kognitiven und emotionalen Wirkung von Medien-Frames

Randbedingungen kognitiver Wirkungen

z.B. Vorwissen und Voreinstellungen

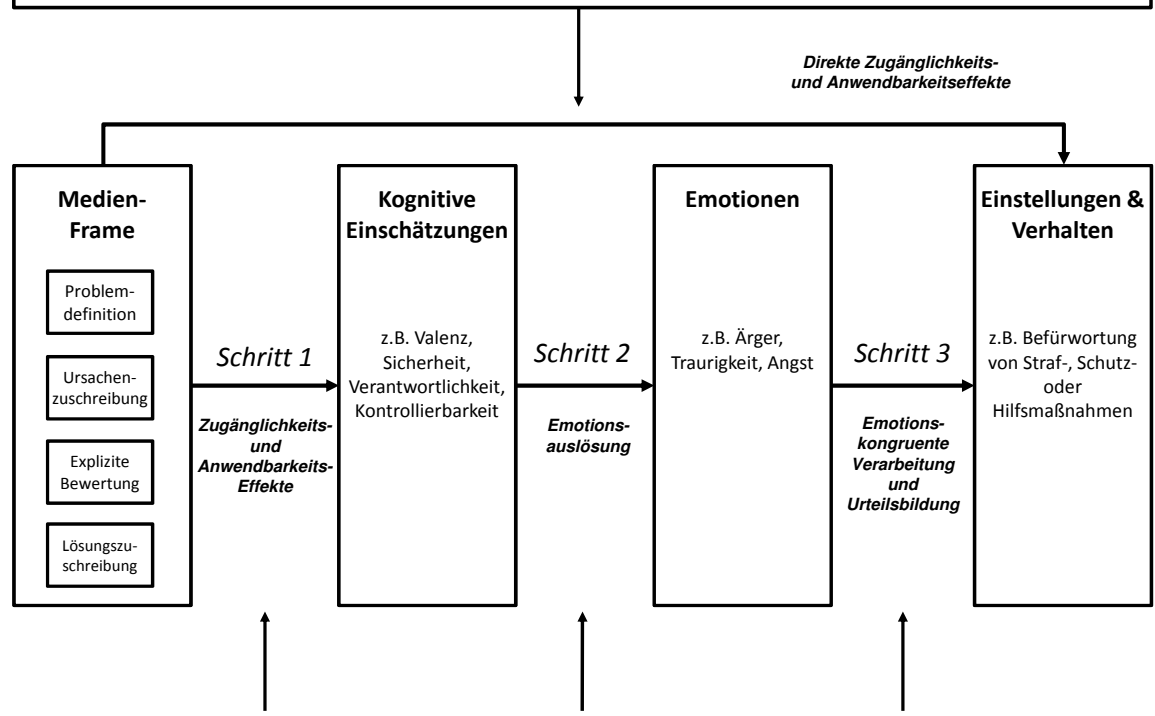

Randbedingungen der Auslösung und Wirkung von Emotionen

z.B. Voreinstellungen, emotionale Neigung

Befunde einer ersten Studie deuten darauf hin, dass emotionale Framing-Effekte tatsächlich dreistufige Prozesse darstellen. Kühne und Schemer (2013) stellten fest, dass die Manipulation der Ursachenzuschreibung für Verkehrsunfälle in einem Nachrichtenbeitrag einen indirekten Einfluss auf die Einstellung der Rezipienten zu Strafmaßnahmen hatte: Wenn die Schuld für einen Verkehrsunfall einem Autofahrer zugeschrieben wurde, führte dies zu höheren Einschätzungen personaler Verantwortlichkeit für den Unfall. Die höhere Einschätzung personaler Verantwortlichkeit führte in einem zweiten Schritt zu stärkerem Ärger, der wiederum die Zustimmung zu Strafmaßnahmen erhöhte. Die Wirkung der Frame-Manipulation auf die Einstellung zu Strafmaßnahmen wurde also über kognitive Einschätzungen und ausgelöste Emotionen vermittelt.

Das Modell ergänzt bisherige Theorien, die Framing-Effekte ausschließlich durch kognitive Prozesse erklären. Zwar dürften viele Medien-Frames Einstellungen direkt über kognitive Zugänglichkeits- und Anwendbarkeitseffekte beeinflussen. Wenn diese kognitiven Prozesse aber zur Aktivierung emotionsspezifischer Einschätzungsmuster führen, dann können Frames auch Emotionen auslösen. Die Wirkung einer Untermenge von Medien-Frames wird demnach durch Kognitionen und Emotionen vermittelt. Der relative Beitrag von Kognitionen und Emotionen zur Urteilsbildung sollte dabei von ihrem Aktivierungsgrad abhängig sein: Je stärker die ausgelösten Emotionen sind, desto größer ihr Wirkpotenzial bei der Urteilsbildung. Dabei beeinflussen verschiedene Moderatoren, ob kognitive oder emotionale Reaktionen überwiegen. 
$\mathrm{Ob}$ ein Medien-Frame primär Kognitionen oder Emotionen aktiviert, ist von Bedeutung, da kognitive und emotionale Prozesse oft unterschiedliche Resultate hervorbringen. Erstens führen emotionale Prozesse im Vergleich zu kognitiven Prozessen zu stärkeren, extremeren und besser zugänglichen Einstellungen. Zweitens gilt zu berücksichtigen, dass auch der Effekt von Emotionen und Kognitionen auf Einstellungen durch Moderatoren beeinflusst wird. Deshalb können je nach Rezeptionssituation (d. h.: je nachdem, wie die Moderatoren ausgeprägt sind) entweder Kognitionen oder Emotionen eine stärkere Wirkung auf die Einstellungen der Rezipienten haben. Bei Rezipienten mit einer starken Neigung, Urteile auf Emotionen aufzubauen, dürften emotionale Reaktionen zum Beispiel zu einer stärkeren Einstellungsänderung führen als kognitive Reaktionen.

Für die empirische Untersuchung von Framing-Effekten empfehlen wir deshalb, dass Codebücher für Inhaltsanalysen so gestaltet werden, dass Frame-Konfigurationen erfasst werden können, die Emotionen auslösen sollten. Ebenso sollten Fragebögen zur Untersuchung von Framing-Effekten die emotionalen Reaktionen der Rezipienten sowie die zentralen Moderatoren emotionaler Prozesse messen. Die Berücksichtigung dieser Messungen erlaubt es, die Wirkungen von Medien-Frames auf Einstellungen genauer zu modellieren.

\section{Diskussion}

Im vorliegenden Beitrag wurde ein integratives Modell für die Erklärung von FramingEffekten auf Einstellungen von Rezipienten skizziert. Erste empirische Befunde deuten darauf hin, dass Medien-Frames Einstellungen und Verhaltensintentionen tatsächlich über die Auslösung von Emotionen beeinflussen können (Kühne \& Schemer, 2013). Theoretische Erweiterungen und empirische Überprüfungen des Modells sind aber erforderlich.

Erstens sollte erörtert werden, wie Medien-Frames aufgebaut sein müssen, um andere Emotionen als Ärger und Traurigkeit auszulösen, und welche Wirkung diese Emotionen haben. Von besonderem Interesse sind dabei der Aufbau und die Wirkung von MedienFrames, die positive Emotionen wie Stolz und Hoffnung auslösen, da diese Emotionen in der Persuasionsforschung bislang kaum beachtet wurden.

$\mathrm{Zu}$ überprüfen ist weiterhin, welche emotionale Wirkung Medien-Frames haben, die nicht die Struktur idealtypischer Emotions-Frames besitzen. Dies ist von Bedeutung, da in der Medienberichterstattung oft keine idealtypischen Emotions-Frames vorliegen dürften. Zu klären ist zum Beispiel die Frage, ob Medien-Frames Emotionen auslösen, wenn gewisse Frame-Komponenten fehlen. Ebenso ist die emotionale Wirkung von Nachrichtenbeiträgen zu untersuchen, die Komponenten verschiedener emotionaler Frames kombinieren. Anzunehmen ist, dass solche Misch-Frames beim Rezipienten Mischemotionen auslösen können, die mit spezifischen Wirkungen auf Einstellungen verbunden sind.

Notwendig ist auch eine Überprüfung der Annahme, dass Medien-Frames sowohl bei einer oberflächlichen als auch einer intensiven Informationsverarbeitung Emotionen auslösen können. Emotionen wird oft die Rolle von Heuristiken zugeschrieben, auf die bei einer oberflächlichen Informationsverarbeitung zurückgegriffen wird. Gemäß unserem Modell sollten Emotionen indessen auch bei einer intensiven Verarbeitung von Medien-Frames Emotionen auslösen, indem sie die Anwendbarkeit von kognitiven Einschätzungen erhöhen. Zu überprüfen ist in diesem Zusammenhang insbesondere, ob in Abhängigkeit von der Verarbeitungsweise unterschiedliche Moderatoren die Stärke der emotionalen Effekte beeinflussen. Denkbar ist zum Beispiel, dass der Rezipient bei einer 
intensiven Verarbeitung eher die Möglichkeit zur Emotionsregulation besitzt und Emotionseinflüsse gezielt unterbinden kann.

Eine ausführliche Untersuchung der emotionalen Prozesse sollte die Präzision von Framing-Modellen erhöhen, die bislang ausschließlich kognitive Prozesse postulieren. Diese Präzision ist nicht nur aus analytischen, sondern auch aus normativen Gesichtspunkten von Bedeutung: Emotionale Effekte auf Meinungsbildungsprozesse sind oft unerwünscht. Die Überprüfung eines Modells emotionaler Framing-Effekte kann Hinweise liefern, unter welchen Bedingungen emotionale Wirkungen von Medienberichten erwartet werden können und wie diesen Wirkungen begegnet werden kann.

\section{Literatur}

Bryant, J., Roskos-Ewoldsen, D., \& Cantor, J. (Hrsg.). (2003). Communication and Emotion: Essays in Honor of Dolf Zillmann. Mahwah, NJ: Erlbaum.

Cho, H., \& Boster, F. J. (2008). Effects of Gain versus Loss Frame Antidrug Ads on Adolescents. Journal of Communication, 58, 428-446.

Chong, D., \& Druckman, J. N. (2007). Framing Public Opinion in Competitive Democracies. American Political Science Review, 101(4), 637-655.

Druckman, J. N., \& Nelson, K. R. (2003). Framing and Deliberation: How Citizens' Conversations Limit Elite Influence. American Journal of Political Science, 47(4), 729-745.

Eagly, A. H., \& Chaiken, S. (1993). The Psychology of Attitudes. Fort Worth, TX: Harcourt Brace Jovanovich.

Entman, R. M. (1993). Framing: Toward Clarification of a Fractured Paradigm. Journal of Communication, 43(4), 51-58.

Forgas, J. P. (1995). Mood and Judgment: The Affect Infusion Model (AIM). Psychological Bulletin, 117(1), 39-66.

Gamson, W. A., \& Modigliani, A. (1987). The Changing Culture of Affirmative Action. In R. G. Braungart \& M. M. Braungart (Hrsg.), Research in Political Sociology. A Research Annual (Vol. 3, S. 137-177). Greenwich, CT: JAI Press.

Gamson, W. A., \& Modigliani, A. (1989). Media Discourse and Public Opinion on Nuclear Power: A Constructionist Approach. American Journal of Sociology, 95(1), 1-37.

Gross, K. (2008). Framing Persuasive Appeals: Episodic and Thematic Framing, Emotional Response, and Policy Opinion. Political Psychology, 29(2), 169-192.

Gross, K., \& Brewer, P. R. (2007). Sore Losers: News Frames, Policy Debates, and Emotions. Press/ Politics, 12(1), 122-133.

Gross, K., \& D’Ambrosio, L. (2004). Framing Emotional Response. Political Psychology, 25(1), $1-29$.

Han, S., Lerner, J. S., \& Keltner, D. (2007). Feelings and Consumer Decision Making: The Appraisal-Tendency-Framework. Journal of Consumer Psychology, 17(3), 158-168.

Harmon-Jones, E., Harmon-Jones, C., Amodio, D. M., \& Gable, P. A. (2011). Attitudes toward Emotions. Journal of Personality and Social Psychology, 101(6), 1332-1350.

Iyengar, S. (1991). Is Anyone Responsible? How Television Frames Political Issues. Chicago, IL: University of Chicago Press.

Kim, H. J., \& Cameron, G. T. (2011). Emotions Matter in Crisis: The Role of Anger and Sadness in the Publics' Response to Crisis News Framing and Corporate Crisis Response. Communication Research, 38(6), 826-855.

Kühne, R., \& Schemer, C. (2013, im Druck). Emotionale Framing-Effekte auf politische Einstellungen und Partizipationsbereitschaft. In F. Marcinkowski (Hrsg.). Framing als politischer Prozess. Aufsätze zur Politischen Kommunikation entwickelter Demokratien. Baden-Baden: Nomos.

Lazarus, R. S. (1991). Emotion E Adaptation. Oxford: Oxford University Press.

LeDoux, J. E. (1999). The Emotional Brain: The Mysterious Underpinnings of Emotional Life. London: Phoenix. 
Lerner, J. S., \& Keltner, D. (2000). Beyond Valence: Toward a Model of Emotion-Specific Influences on Judgment and Choice. Cognition and Emotion, 14(4), 473-493.

Leventhal, H., \& Scherer, K. (1987). The Relationship of Emotion to Cognition: A Functional Approach to a Semantic Controversy. Cognition and Emotion, 1(1), 3-28.

Maio, G. R., \& Esses, V. M. (2001). The Need for Affect: Individual Differences in the Motivation to Approach or Avoid Emotions. Journal of Personality, 69(4), 583-615.

Marcus, G. E. (2002). The Sentimental Citizen: Emotion in Democratic Politics. University Park, PA: The Pennsylvania State University Press.

Matthes, J. (2007). Framing-Effekte: Zum Einfluss der Politikberichterstattung auf die Einstellungen der Rezipienten. München: Verlag Reinhard Fischer.

Miller, P. R. (2011). The Emotional Citizen: Emotion as a Function of Political Sophistication. Political Psychology, 32(4), 575-600.

Nabi, R. L. (2003). Exploring the Framing Effects of Emotion: Do Discrete Emotions Differently Influence Information Accessibility, Information Seeking, and Policy Preference. Communication Research, 30(2), 224-247.

Nelson, T. E., Oxley, Z. M., \& Clawson, R. A. (1997). Toward a Psychology of Framing Effects. Political Behavior, 19(3), 221-246.

Nerb, J. (2000). Die Bewertung von Umweltschäden: Kognitive und emotionale Folgen von Medienmeldungen. Bern: Hans Huber.

Oatley, K., \& Jenkins, J. M. (1996). Understanding Emotions. Cambridge, MA: Blackwell.

Ortony, A., Clore, G. L., \& Collins, A. (1988). The Cognitive Structure of Emotions. Cambridge: Cambridge University Press.

Pan, Z., \& Kosicki, G. M. (1993). Framing Analysis: An Approach to News Discourse. Political Communication, 10, 55-75.

Peter, J. (2002). Medien-Priming: Grundlagen, Befunde und Forschungstendenzen. Publizistik, 47(1), 21-44.

Price, V., \& Tewksbury, D. (1997). News Values and Public Opinion: A Theoretical Account of Media Priming and Framing. In G. A. Barnett \& F. J. Boster (Hrsg.), Progress in Communication Sciences. Vol. 13. Advances in Persuasion (S. 173-212). Greenwich, CT: Ablex Publishing Corporation.

Schenk, M. (2007). Medienwirkungsforschung (3. Auflage). Tübingen: Mohr Siebeck.

Scherer, K. R. (2001). Appraisal Considered as a Process of Multilevel Sequential Checking. In K. R. Scherer, A. Schorr, \& T. Johnstone (Hrsg.), Appraisal Processes in Emotion. Theory, Methods, Research (S. 92-120). New York, NY: Oxford University Press.

Scheufele, B. (2003). Frames - Framing - Framing-Effekte: Theoretische und methodische Grundlegung des Framing-Ansatzes sowie empirische Befunde zur Nachrichtenproduktion. Wiesbaden: Westdeutscher Verlag.

Scheufele, D. A., \& Tewksbury, D. (2007). Framing, Agenda Setting, and Priming: The Evolution of Three Media Effects Models. Journal of Communication, 57, 9-20.

Schmitt, A., \& Mees, U. (2000). Trauer. In J. H. Otto, H. A. Euler, \& H. Mandl (Hrsg.), Emotionspsychologie. Ein Handbuch (S. 209-220). Weinheim: Psychologie Verlags Union.

Shen, L., \& Dillard, J. P. (2007). The Influence of Behavioral Inhibition/Approach Systems and Message Framing on the Processing of Persuasive Health Messages. Communication Research, 34(4), 433-467.

Sonnemans, J., \& Frijda, N. H. (1995). The Determinants of Subjective Emotional Intensity. Cognition and Emotion, 9(5), 483-506.

Way. B. M., \& Masters, R. D. (1996). Emotion and Cognition in Political Information Processing. Journal of Communication, 46(3), 48-65.

Wirth, W., \& Schramm, H. (2005). Media and Emotions. Communication Research Trends, 24(3), 3-39.

Zillmann, D., \& Knobloch, S. (2001). Emotional Reactions to Narratives about the Fortunes of Personae in the News Theater. Poetics, 29, 189-206. 